EPJ Web of Conferences 106, 02009 (2016)

DOI: $10.1051 /$ epjconf/201610602009

(C) Owned by the authors, published by EDP Sciences, 2016

\title{
A History of Dosimetry for the Advanced Gas-cooled Reactors
}

\author{
Simon Shaw ${ }^{1, a}$ and Dean Thornton ${ }^{2}$ \\ ${ }^{1}$ EDF Energy Nuclear Generation Ltd, Gloucester, UK \\ ${ }^{2}$ AMEC, Clean Energy - Europe, Gloucester, UK
}

\begin{abstract}
This paper presents a summary of the methods used in the first $\sim 40$ years of AGR neutron dosimetry and nuclear heating calculations, and the influence of the earlier Magnox reactor dosimetry programme. While the current state-of-the-art Monte Carlo methods are extremely powerful they still require very careful consideration of the quality of the input data, nuclear data validation and variance reduction techniques; in particular, this paper examines the difficulties in assuring the adequate convergence of calculations when Monte Carlo acceleration is applied in the presence of significant streaming paths through attenuating or scattering media.
\end{abstract}

\section{The UK AGR Reactors}

EDF Energy operates 14 Advanced Gas-cooled Reactors (AGR), located in pairs at seven nuclear power stations in the UK. The AGRs are the second generation of nuclear power plants in the UK. Hinkley Point B was the first AGR to start operating (1976), the last was Torness A (1988).

Like the earlier Magnox reactors, AGRs (Fig. 1) use graphite for moderation and pressurised carbon dioxide $\left(\mathrm{CO}_{2}\right)$ as coolant. The AGR system was designed to provide high pressure steam, at high temperatures $\left(\sim 650^{\circ} \mathrm{C}\right)$, to the turbines. With a temperature rise in the boilers of over $300^{\circ} \mathrm{C}$, the AGRs achieve generating efficiencies of $\sim 40 \%$.

An AGR fuel element comprises 36 stainless steel fuel pins, each containing enriched $\mathrm{UO}_{2}$, held within a graphite sleeve (Fig. 1). Eight fuel elements ${ }^{\mathrm{b}}$ are linked axially by a nickel steel tie bar to form a "fuel stringer" for each of the core's fuel channels. The interlinked graphite sleeves provide a pressure boundary between an upward flow cooling the fuel and a downward re-entrant flow cooling the graphite moderator. The AGR designs have differences, but most load $\sim 300$ fuel stringers ${ }^{\mathrm{b}}$.

The graphite core is formed from columns of polyhedral or cylindrical bricks, with a central channel to receive the fuel, arranged on a lattice with pitch $^{\mathrm{b}} 46 \mathrm{~cm}$. The moderator bricks also contain smaller vertical holes to allow the permeation of methane $\left(\mathrm{CH}_{4}\right)$, which is introduced to the $\mathrm{CO}_{2}$ coolant to inhibit graphite oxidation. Control rods and other ancillary devices are introduced via smaller channels located within square "interstitial" bricks ${ }^{b}$ in the gap where the corners of four surrounding fuel

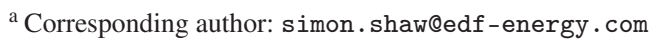

b At the Dungeness B AGRs fuel stringers are formed from seven elements; the lattice of 408 fuel channels has a smaller pitch $(39 \mathrm{~cm})$ and control rods are introduced via vacant channels on the fuel lattice.
}

This is an Open Access article distributed under the terms of the Creative Commons Attribution License 2.0, which permits unrestricted use, distribution, and reproduction in any medium, provided the original work is properly cited. 

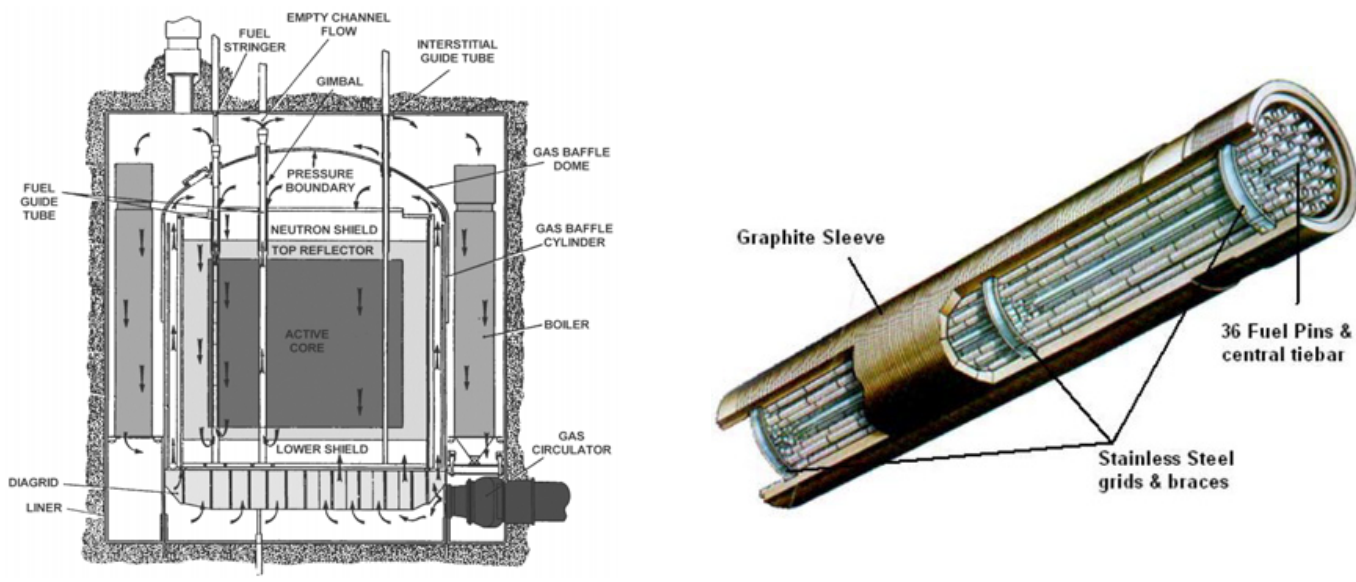

Figure 1. Left: Schematic layout of an AGR reactor (arrows show directions of gas flow); Right: AGR fuel element with part of the graphite sleeve cut away, each fuel element is approximately $1 \mathrm{~m}$ long.

bricks meet. The active core is surrounded by additional columns and layers of graphite bricks that form the radial and axial neutron reflectors and provide additional shielding.

The steel core support structure provides a flat base for the graphite core, fuel and other reactor assemblies, at a level above the reactor pressure vessel liner to allow coolant flow into the base of the fuel channels. The outer bricks of the radial shield are linked via the steel core restraint system to anchor points on the boiler shield wall, which is a massive cylindrical steel wall that also extends up to the gas baffle dome to form a pressure boundary between the inlet and outlet coolant flows.

The whole circuit, including boilers and gas circulators is contained within a pre-stressed concrete pressure vessel. A fuelling machine allows on-load refuelling (at reduced power) via the pile cap.

EDF Energy aims to extend the operating life of all the AGRs.

\section{Dosimetry in the AGR Design and Early Operation}

A fundamental aspiration of the AGR design was to reduce irradiation induced issues that affected the earlier Magnox reactors. Given the order of magnitude increase in power density in the AGRs, features were included to eliminate or minimise the effects of increased irradiation, and radiological dose rates during shutdown, on the basis of a 25 year design life for the AGRs:

- Concrete pressure vessels, due to dosimetry issues affecting early Magnox steel pressure vessels.

- Additional graphite in the side reflector to protect core restraint steel-work.

- Boiler shield wall, reduces activation to a level that permits shut-down man access to the boilers.

- Complex shields to reduce neutron streaming to steel-work above and below the core.

- The "methane holes" to reduce radiolytic oxidation of the graphite bricks.

While graphite sample carriers were installed in the AGR cores, and it was recognised that the high AGR operating temperature would increase the susceptibility of some steel components to creep, a steels surveillance programme was not considered necessary. Few measurements of AGR neutron fluences have been made, other than during commissioning. Calculations performed at that time were for the primary purpose of confirming the design intent and were typically one-dimensional diffusion based methods or bounding approximations. 


\section{Impact of the Magnox Experience on AGR Dosimetry}

As the Magnox reactors approached their design lives, it became apparent that the dosimetry related materials issues were more diverse and challenging than had been originally anticipated. In the late 1980's a ten year programme of measurements, methods developments and dosimetry assessments was begun to support continued operation of the Magnox plant [1]. Some issues affecting Magnox were shown to be relevant and prompted developments to the AGR dosimetry programme.

\subsection{Measurements}

The Magnox reactors were equipped with sophisticated surveillance schemes comprising materials and neutron flux monitors for the measurement of fast neutron irradiation (initially of iron, sulphur and nickel), but it became clear that the surveillance locations were not representative of key locations or the spectrum of neutrons causing embrittlement at assessment locations. In response, extensive additional measurement coverage was provided for each reactor:

- Flux monitors to represent both thermal (n, $\gamma$ ) and fast reactions, including ${ }^{93} \mathrm{Nb}(\mathrm{n}, \mathrm{n}$ ').

- Retrospective measurements on material recovered from the reactors during outage periods.

- Measurements from capsules of flux monitoring materials suspended in key locations (these directly informed ongoing measurements in the Hinkley Point B and Hunterston B AGRs [2]).

\subsection{Calculational Methods and Models}

Very early on, it was realised that due to large variations in neutron spectrum, material damage in steel components and surveillance specimens could only be correlated satisfactorily against doses characterised by calculations of a mechanistically derived dose parameter. The aim was then to provide the neutron irradiation, in displacements per atom (dpa), for every surveillance specimen and assessment location.

Three-dimensional radiation transport models were required to adequately represent neutron streaming through volumes of gas in the complicated Magnox geometries; these were developed using the UK Monte Carlo neutron transport code MCBEND [3] and were also used to to underwrite the predictions of the models by validation against the measurements (Sect. 3.1). Although spectrum adjustment techniques were never used, satisfactory agreement for a range of locations and energy dependencies was used as evidence that the models predicted without significant bias. Where evidence existed for systematic uncertainties, dpa recommendations were scaled by mean Calculated to Experimental $(C / E)$ values.

\subsection{Uncertainties Derived from Benchmarks and Later Measurements}

While comparisons with plant measurements provided strong evidence for the abilities of the models to predict neutron fluxes in a diverse range of locations, the performance in the region $\sim 0.1-2 \mathrm{MeV}$ (i.e. that which contributes most strongly to dpa) was not as good. From the results of fast neutron reactions ${ }^{32} \mathrm{~S}(\mathrm{n}, \mathrm{p}){ }^{115} \mathrm{In}\left(\mathrm{n}, \mathrm{n}^{\prime}\right)$ and ${ }^{103} \mathrm{Rh}(\mathrm{n}, \mathrm{p})$, the Winfrith graphite benchmark comparison [4] indicated that MCBEND would systematically and progressively under-predict fast dpa at graphite penetrations exceeding $50 \mathrm{~cm}$; this was confirmed with measurements of ${ }^{93} \mathrm{Nb}\left(\mathrm{n}, \mathrm{n}^{\prime}\right)$ on the plant (see Fig. 2 of [1]). Modelling of the benchmarks with MCNP and a range of nuclear data libraries showed this to be a generic effect and attributed to deficiencies in the nuclear data for carbon (e.g. see [1]). The issue was addressed by the application of an empirically derived correction; the Nuclear Data Correction Factor 

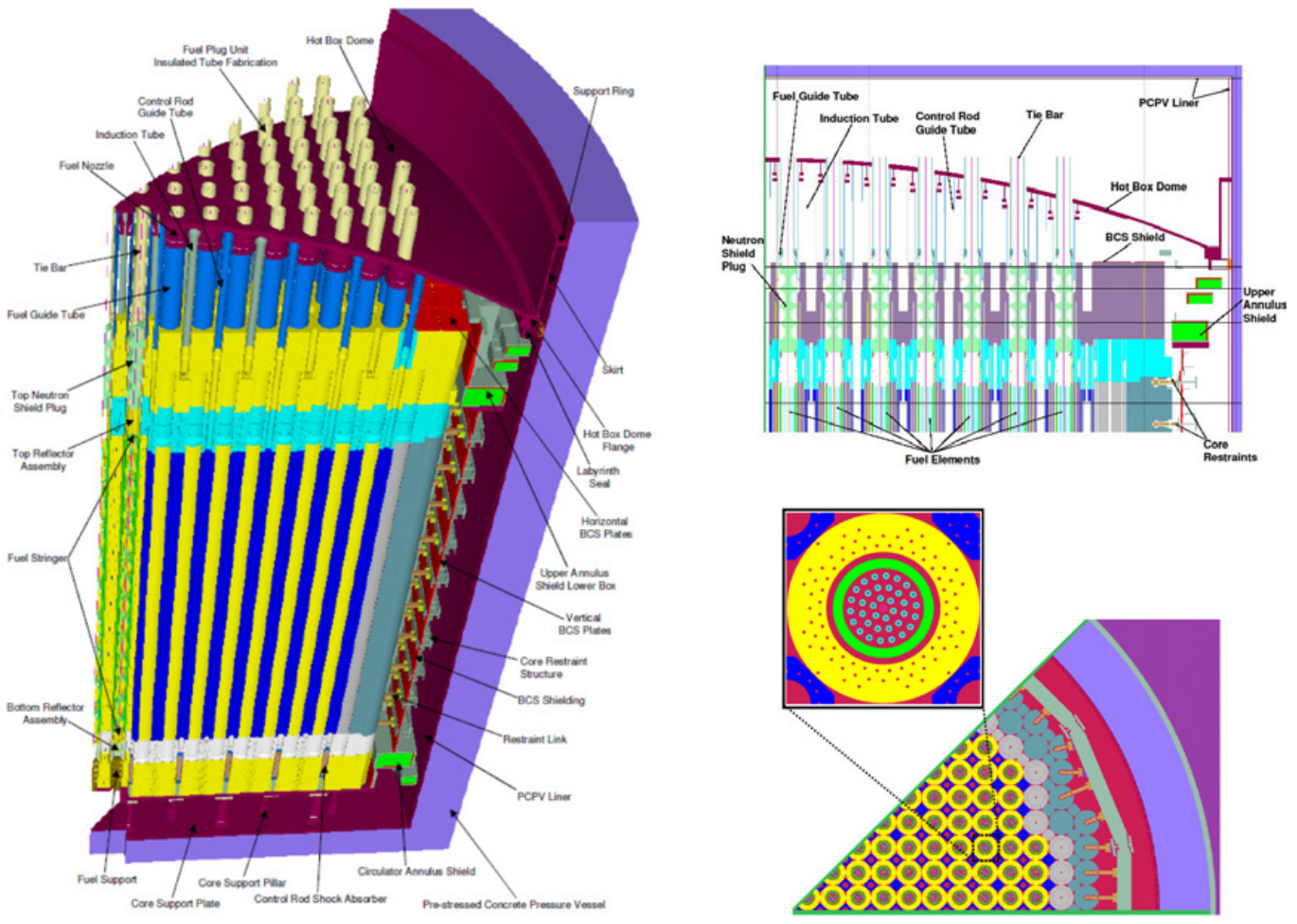

Figure 2. Left: MCBEND AGR core octant model; Top right: detail of the top-core region along the $45^{\circ}$ radial plane; Bottom Right - horizontal plane through the core mid-height with an inset showing details of a fuel channel.

(NDCF) varies linearly from 1.0 at $50 \mathrm{~cm}$ thickness (i.e. no observed effect) to 1.43 at a maximum thickness of $71.6 \mathrm{~cm}$ beyond which there are no data [1].

This issue is encountere in the AGR dosimetry programme where there are structural components behind graphite thicknesses well in excess of $71.6 \mathrm{~cm}$. These are discussed later.

\subsection{Thermal and Fast Neutrons}

During the mid-1990s the potential for thermal neutrons to cause embrittlement in steel was recognised. The same calculational route was extended to recommend dpa rates for neutrons with energy $<1 \mathrm{keV}$, for both surveillance specimens and assessment locations. Since the physical mechanisms of the displacement of iron atoms by fast neutrons (i.e. elastic and inelastic collisions) and thermal neutrons (i.e. atomic recoil following $\gamma$-ray emission) are entirely different, the two calculations were reported separately as "fast dpa" ( $E>1 \mathrm{keV})$ and "thermal" dpa $(E<1 \mathrm{keV})$.

In Magnox reactors large variations (50-100\%) in the relative importance of thermal neutrons were evident, primarily dependent on the thickness of local graphite. Experiments in the strong thermal flux of the Halden heavy water reactor demonstrated greater rates of predicted steels properties changes per unit dpa from thermal neutrons than from fast neutrons. Consequently, a thermal neutron effectiveness factor " $k$ " was empirically derived to enable total displacement damage $=\mathrm{dpa}_{\mathrm{fast}}+k \mathrm{dpa}_{\text {thermal }}$ to be used in the correlation and assessment of steels irradiated properties. Values of $k$ within the range 1.5 to 2.5 for C-Mn steel plate and welds are applied to AGR assessments, which use the same ferritic steels properties as the Magnox reactors [5]. 
As introduced by Magnox, fast and thermal dpa rates (dpa/GWd) for all locations required for AGR structural integrity assessments are published via a single verified source, the Dosimetry Data Manual. The DDM is regularly updated and endorsed by an independent panel, allowing structural integrity experts to easily locate quality assured and recommended dose data for use in their assessments without them requiring a detailed understanding of the dosimetry methods.

\subsection{Nuclear Heating}

Early on in the Magnox programme, it was recognised that increased irradiation temperature reduced the embrittling effect of neutron irradiation in ferritic steels and a temperature dependent correction was included in the trend curves as a result. Nuclear heating due to neutron and gamma radiation makes an important contribution to the elevation of component temperatures above ambient values.

Nuclear heating in steel is greatest where thermal neutron fluxes are high and in the AGRs this is particularly important in the side-core restraint regions where the energy deposition rate is dominated by gamma rays from local $(n, \gamma)$ reactions. The ability of MCBEND to perform coupled $(n, \gamma)$ calculations was validated against the Winfrith Capture Gamma-ray benchmark study carried out in the ASPIS facility [6]. Damage to steel from gamma irradiation is likewise dominated by the local flux from (n, $\gamma)$ reactions. Other benchmark comparisons have also been made to validate the ability of MCBEND to reproduce the transport of gamma rays through iron [7], and the energy deposition in graphite from a mixed (neutron, fuel gamma and capture gamma) radiation field [8].

\section{Modern AGR Dosimetry Calculations with MCBEND}

Modern calculations in the UK use the MCBEND Monte Carlo radiation transport code [3] for direct 3-D calculations (or reconstruction of volumetric data from 2-D calculations [9]). The whole reactor core is modelled to ensure that all significant routes that neutrons may take through the complicated geometry are represented (in practice the core symmetry allows an octant with reflecting boundaries).

Variance reduction techniques are required to achieve satisfactory convergence of the calculation. MCBEND's CALCULATE option is used to calculate importance maps on a user defined $(x, y, z)$ or $(r, \theta, z)$ mesh via an initial adjoint neutron diffusion calculation using the required scoring response function (e.g. dpa) as a source. Using these maps, the forward Monte Carlo calculations are accelerated by using source weighting and/or the splitting/Russian-roulette techniques.

A number of facilities allow the user to assess the statistical reliability of the results: scoring interim results; checking for undue increases in variance or variance of variance; scoring weight distributions of scoring samples with percentage contributions to the result from each weight bin. MCBEND11 also allows the option of applying statistical tests to give a degree of automation to the analysis of the statistical reliability. The relative performances of MCBEND and MCNP have also been compared against benchmark experiments and found to give consistent results when using the same nuclear data libraries, noting that both codes show similar under-predictions of lower energy fast neutron responses in graphite for all nuclear data libraries as noted in Sect. 3.3.

For lifetime damage dose assessments a realistic neutron source is provided by the PANTHER reactor physics code [10], which is applied to logs of plant thermal conditions to reconstruct histories of reactor power, fuel channel ratings and also to provide axial power profiles.

The large size of AGR reactors (>10 m diameter) and the combination of regularly arranged channels with curved features and components on a range of scales makes modelling them a challenging task (see Fig. 2). In addition to a lack of international experience and benchmarking data, there are a number of generic issues particular to AGRs that require special consideration. 


\subsection{Graphite Moderated Reactors}

The key nuclear safety functions of the graphite moderator are to maintain coolant flow and to ensure free movement of the fuel and control rods at all times, including under fault scenarios. Hence the graphite moderator, which can not be replaced or repaired, is a life-limiting component of the reactor.

Irradiation leads to dimensional change of the graphite bricks [11]. Initially the graphite shrinks causing mechanical stress and the possibility for the bricks to crack; after further irradiation (operation $\sim 18 \mathrm{TWd}$ ) the graphite is expected to swell causing differential stresses and the potential for cracks to

traverse the full width of the brick. Sufficient numbers of cracked bricks potentially challenge the ability of the core to perform its nuclear safety function.

Energy deposition by radiation in the bricks also drives moderator Graphite Weight Loss (GWL). Radiolytic oxidation of the $\mathrm{CO}_{2}$ coolant forms oxygen free radicals that react quickly with the carbon in the graphite bricks to form carbon monoxide, which is then lost via the coolant flow. It is predicted that GWL exceeding $40 \%$ could be experienced in localised positions by the end of the plant operating life time with consequent effects on the reactor physics parameters of the core and the leakage of neutrons outside the core as the number density of the moderator reduces.

Dosimetry calculations of the effects of prolonged exposure of the graphite bricks to irradiation damage and nuclear energy deposition are thus very important to support the ongoing graphite core safety cases and plant life extension decisions and have been described previously [9].

\subsection{Reactor Physics Effects}

The thermal neutron flux increases in the AGR reflector region, in the absence of any fuel to absorb the leaking thermal neutrons. Coupled with the low neutron capture cross-section of carbon, low energy neutrons are a significant, if not the dominant, contributor to the total steel damage for excore components, especially in regions beyond the neutron reflector: for example in AGR core-restraint links the thermal dpa is a factor of 3-4 higher than the fast dpa. Therefore it is generally necessary to perform Monte Carlo dosimetry calculations for fast and thermal dpa in AGRs separately, with variance reduction schemes individually optimised for each.

AGRs have several banks of control rods which are used for bulk reactivity control and flux shaping, and each AGR operates with different rod insertion strategies. Although it is possible to extract the control rod positions from the station "core follow" logs, MCBEND is used as a "fixed source" code and it is not practical to incorporate reactivity feedback effects on the neutron population. In any case the PANTHER core follow route ensures that the effects of the control rods on reactor power are incorporated in the source normalisation to give dose rates in $\mathrm{dpa} / \mathrm{GWd}$. Generally the omission of control rods is conservative as it under represents the absorption in the system, although this neglects a component of heating from secondary $\gamma$-rays from the control rods.

\subsection{Neutron Streaming}

The fast neutron dose to ex-core components is limited by the design of the AGR core graphite shielding. Hence Monte Carlo variance reduction techniques are required to overcome this large attenuation and produce meaningful ex-core doses. However, the use of $\mathrm{CO}_{2}$ as coolant means that gaps between graphite components are essentially non-attenuating, in particular the vertical channels containing the fuel or the control rods, but also gaps between adjacent graphite columns and the methane holes. The boundaries of these streaming paths are not well represented in $(x, y, z)$ or $(r, \theta, z)$ geometries and may intersect individual cells of the importance maps. While the importance map may be optimised to maintain the population of particles travelling through graphite, particles with parallel trajectories in the coolant may be split many times by the variance reduction scheme with very few, if any, fragments 

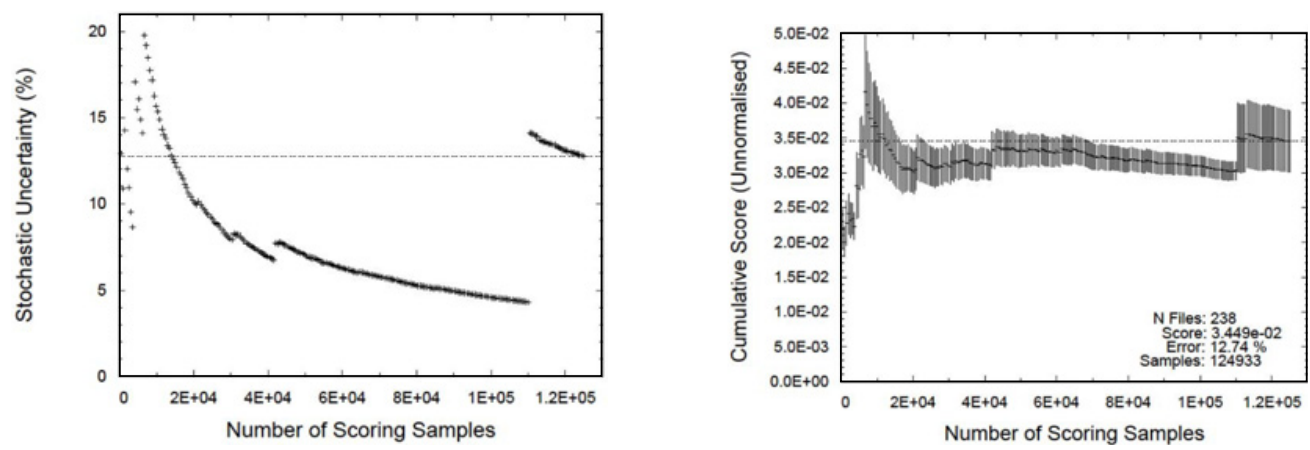

Figure 3. Convergence of a Monte Carlo dpa dose calculation for an AGR component. Note how the dose seems to be converging to an uncertainty $\sim 4 \%$ before being disrupted by a high weight event.

lost by attenuation. At best this results in an inefficient calculation that can take many hours to track a very large number of low-weight particle fragments from an initial high-weight source particle. These very long particle histories are also at greater risk of overrunning imposed time limits and being removed from the calculation, which could bias the results against these low probability but high transmission routes through the problem geometry.

For problems where the bulk transport of radiation is likely to be in the same direction as the predominantly vertical streaming paths (i.e. for doses to AGR gas baffle dome, or core support) particles taking these routes may make a significant contribution to the mean score. While there may be a very small probability of a particle to enter one of these narrow pathways, there is very little attenuation along it and hence a very high probability that the particle will not only reach the scoring location but may also retain most of its initial energy.

\section{Practical Experiences of Whole Core AGR Dosimetry Calculations}

This final section discusses practical experiences of some of the issues noted above that were encountered during a two-year programme of work to produce a full 3-dimensional MCBEND model of one of the AGR reactor designs and calculate doses to ex-core structural components.

\subsection{Living with Streaming Particles}

Fast and thermal dpa calculations were conducted for sub-core, side-core and top-core regions separately. To maximise available computing resources, and to have confidence that the solution had achieved convergence, many individual calculations were performed for each core region and combined via a statistically robust method.

Some results had a highly disruptive effect on the convergence of the mean (Fig. 3). It was tempting to remove these data as "outliers", but this would be incorrect: in fact these data indicate that the Monte Carlo solution may not be well sampled and that the calculated dosimetry is unreliable. These events take real, but rare, routes through the model geometry, as noted in Sect. 4.4.

Fundamentally, the convergence of these results can only be improved by increasing the size of the statistical sample (increasing number of calculations) or modifying the importance map, but this may not be practical. Instead a simple method was used to assess the confidence in the stochastic uncertainty reported by MCBEND: as each calculation completed, the results were combined into a single global average. If the global stochastic uncertainty was $<15 \%$ then the calculation with largest value of the second moment, $\sum\left(x^{2}\right)$, was identified. The degree of convergence was then estimated by calculating 
the percentage change that would be observed in the final score if the identified calculation was counted twice. If the modulus of this value was much less than the final stochastic uncertainty then the calculation was assumed to have converged. A check is also made by comparing with doses at similar locations; large differences indicate that the streaming component is under-sampled.

\subsection{Validity of Fast Neutron Doses}

To deal with the issues of the graphite nuclear data validation limit for fast neutrons (Sect. 3.3), a method was developed based on [12] that used perturbation theory (MCBEND's SENSITIVITY function) to correlate the thickness of material traversed to the sensitivity (first derivative) of the scored responses to the density of graphite materials in the model. The sensitivities from many calculations were combined via a weighted mean and were then compared against similar results from models of the Winfrith single material graphite benchmark. By combining sensitivities in this way, the effects of differing graphite densities (including voids) could be removed and the effective length of graphite material traversed in the model related to actual measurements in the benchmark. This allowed the NDCF to be applied to dpa assessments with effective graphite penetration within the data validation limit of $71.6 \mathrm{~cm}$; where results showed a greater penetration of graphite, bounding results from nearby locations within the validation limit were given. Note that the first analysis of measurements currently being conducted in reactors at Hinkley Point B and Hunterston B are due in early 2015 and will improve the validation data for core restraint doses and may, in the future, extend the validation data beyond $71.6 \mathrm{~cm} \mathrm{[2].}$

Additionally, multiplying the sensitivities by estimates of material density variations (i.e. tolerances on construction material manufacturing specifications) allows a contribution to the total dosimetry uncertainty due to modelling approximations to be estimated.

\subsection{Other Issues Affecting Doses to Locations Above the Core}

Dosimetry above the core is particularly susceptible to the issues noted in the previous sections due to the orientation of the streaming paths and the large thickness of the upper neutron shield $(\sim 2 \mathrm{~m})$.

The AGR core height is expected to reduce over life as the graphite bricks shrink. Since the fuel is supported from the bottom and does not shrink much over its dwell, the shielding above the fuel is reduced as the core height reduces. A mean over life shrinkage in the region of $10-15 \mathrm{~cm}$ is expected, and was found to lead to an increase in dose of up to $75 \%$ in some locations above the core.

Calculations for Magnox found that although the $\mathrm{CO}_{2}$ coolant is a modest contributor to the total neutron attenuation, small effects could be seen due to changes in the coolant density with temperature. The different cooling flows within the AGRs mean that a core averaged $\mathrm{CO}_{2}$ temperature is appropriate for most AGR dosimetry applications. However, above the core, cooler re-entrant flow gas $\left(\sim 300^{\circ} \mathrm{C}\right)$ is separated from the hot boiler feed gas $\left(\sim 650^{\circ} \mathrm{C}\right)$ by the gas baffle dome (see Fig. 1). Sensitivity calculations of the effect of $\mathrm{CO}_{2}$ temperature on doses above the gas baffle dome (high temperature / low density $\mathrm{CO}_{2}$ ) of the Hartlepool A and Heysham 1 AGRs showed that using a core average $\mathrm{CO}_{2}$ density could lead to underestimates in calculated dpa of up to $27 \%$.

\section{Conclusions}

Early experience from the Magnox programme was used to inform the AGR design. The dosimetry programmes for both reactor types developed as understanding of the effects of irradiation on the Magnox materials increased. As experience grew, and aspirations for AGR life extension developed, it became necessary to revisit some of the old design calculations with newer methods in order to quantify, and in some cases reduce, the level of conservatism in the dosimetry judgements. Detailed three-dimensional Monte Carlo calculations, in addition to validation measurements currently being 
conducted in reactors at Hinkley Point B and Hunterston B, will support EDF Energy's considerations of the impacts of dosimetry on the continued safe operation of the AGRs and aspirations for plant life extension.

SS thanks EDF Energy co-workers Geoff Whiley and Paolo Mason for their contributions, and EDF Energy for funding symposium attendance.

\section{References}

[1] T.A. Lewis and D.A. Thornton, "A Decade of Dosimetry for Magnox Reactor Plants", Proc. ISRD-11, Belgium 2002, World Scientific, pp. 269-277 (2003)

[2] D.A. Allen, S.E. Shaw, A.P. Huggon, R.J.Steadman, D.A. Thornton and G.S. Whiley, "Neutron Flux measurements in the side-core region of Hunterston B Advanced Gas-cooled Reactor", Proc. ISRD-14, USA 2011, J ASTM Intl., Vol. 9, No. 3. doi:10.1520/JAI104046 (2012)

[3] P. Cowan, E. Shuttleworth, A.J. Bird and A.J. Cooper, "The Launch of MCBEND 10", Proc. 10th International Conference on Radiation Shielding (ICRS-10) and 13th Topical Meeting on Radiation Protection and Shielding (RPS-2004), Funchal, Madeira Island, Portugal (2004)

[4] S.J. Chucas, W.V. Wright, "MCBEND Validation Report No.2: The Single Material Graphite Benchmark", ANSWERS/VALIDATION/MCBEND/2/1 (1996)

[5] R.B. Jones, D. Edens and M.R. Wootton, "Update on the influence of thermal neutron on the irradiation hardening and embrittlement of plate steels", Proc. IGRDM-8, Nashville USA (1999)

[6] D.J. Picton, "MCBEND Validation Report No.7: ASPIS Capture Gamma Ray Study", ANSWERS/VALIDATION/MCBEND/7/1 (2007)

[7] J.M. McGuiness, S. Newbon, S.J. Chucas, "MCBEND Validation Report No.4: Gamma-Ray Penetration through an Iron Shield", ANSWERS/VALIDATION/MCBEND/4/1 (1994)

[8] H.F. Locke, "MCBEND Validation Report No.9: Energy Deposition in the NESSUS Thimble of the NESTOR Reactor", ANSWERS/VALIDATION/MCBEND/9/1 (1994)

[9] D.O. Morgan A.T. Robinson, D.A. Allen, D.J. Picton, D.A Thornton, S.E. Shaw, "A ThreeDimensional Methodology for the Assessment of Neutron Damage and Nuclear Energy Deposition in Graphite Components of Advanced Gas Cooled Reactors", Proc. ISRD-14, USA 2011, J ASTM Intl., Vol. 9, No. 4. doi:10.1520/JAI104002 (2012)

[10] M. Knight, P. Paul Bryce and Sheldon Hall," WIMS/PANTHER Analysis of UO2/MOX Cores Using Embedded Supercells", Proc. PHYSOR 2012, USA, Adv. Reac. Phys. 1, 77 (2012)

[11] J. Kendall (ed.), "Irradiation damage in graphite due to fast neutrons in fission and fusion systems", IAEA-TECDOC-1154 (2000).

[12] D.A. Thornton, Factors Affecting Predicted Neutron Dose Rates to Steel Pressure Vessels of Magnox Plant, Proc. ISRD-11, Japan 1999, ASTM STP 1398 (2001) 